\title{
TRANSITIONAL SUBJECTS
}




\title{
NEW DIRECTIONS IN CRITICAL THEORY
}

\author{
AMY ALLEN, GENERAL EDITOR
}

New Directions in Critical Theory presents outstanding classic and contemporary texts in the tradition of critical social theory, broadly construed. The series aims to renew and advance the program of critical social theory, with a particular focus on theorizing contemporary struggles around gender, race, sexuality, class, and globalization and their complex interconnections.

For a complete list of books in the series, see page $27 \mathrm{I}$. 


\title{
TRANSITIONAL SUBJECTS
}

\section{CRITICAL THEORY AND OBJECT RELATIONS}

\author{
EDITED BY \\ AMY ALLEN AND \\ BRIAN O'CONNOR
}

Columbia University Press New York 


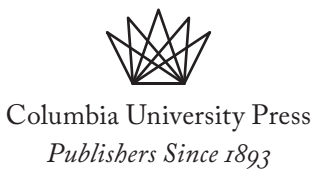

New York Chichester, West Sussex

cup.columbia.edu

\section{Copyright (C) 2019 Columbia University Press}

All rights reserved

Library of Congress Cataloging-in-Publication Data

Names: Allen, Amy, editor. | O’Connor, Brian, I965- editor.

Title: Transitional subjects : critical theory and object relations /

edited by Amy Allen and Brian O'Connor.

Description: New York : Columbia University Press, [2019] | Series: New

directions in critical theory | Includes bibliographical references and index.

Identifiers: LCCN 201804896I| ISBN 9780231183185 (cloth : alk. paper)|

ISBN 9780231183192 (pbk. : alk. paper)| ISBN 9780231544788 (ebook)

Subjects: LCSH: Critical theory. | Object relations (Psychoanalysis) |

Psychoanalysis and philosophy.

Classification: LCC HM480.T73 2019 | DDC 150.19/5-dc23

LC record available at https://1ccn.loc.gov/201804896I

Columbia University Press books are printed on permanent and durable acid-free paper.

Printed in the United States of America

Cover design: Chang Jae Lee

Cover image: Francesca Woodman, Providence, Rhode Island, from Space 2, 1976 copyright (C) 2018 Estate of

Francesca Woodman/Charles Woodman/ARS, New York 